\title{
Risk Preference and Survival Dynamics
}

\author{
Shu-Heng Chen ${ }^{1}$ and Ya-Chi Huang ${ }^{2}$ \\ 1 AI-ECON Research Center \\ Department of Economics \\ National Chengchi University \\ Taipei, Taiwan \\ chchenonccu.edu.tw \\ 2 AI-ECON Research Center \\ Department of Economics \\ National Chengchi University \\ Taipei, Taiwan \\ yachi@aiecon.org
}

Summary. Using an agent-based multi-asset artificial stock market, we simulate the survival dynamics of investors with different risk preferences. It is found that the survivability of investors is closely related to their risk preferences. Among the eight types of investors considered in this paper, only the CRRA investors with RRA coefficients close to one can survive in the long run. Other types of agents are eventually driven out of the market, including the famous CARA agents and agents who base their decision on the capital asset pricing model.

Key words: Market selection hypothesis, Agent-based artificial stock markets, Autonomous agents, Genetic algorithms

\section{Introduction}

The paper is concerned with a part of the debate on the market selection hypothesis. The debate, if we trace its origin, started with the establishment of what become known as the Kelly criterion ([8]), which basically says that a rational long-run investor should maximize the expected growth rate of his wealth share and, therefore, should behave as if he were endowed with a logarithmic utility function. Alternatively speaking, the Kelly criterion suggests that there is an optimal preference (rational preference) which a competitive market will select and that is logarithmic utility. The debate on the Kelly criterion has a long history, so not surprisingly, there is a long list of both pros and cons standing alongside the developments in the literature. ${ }^{3}$

${ }^{3}$ See [11] for a quite extensive review. 
The Kelly criterion may further imply that an agent who maximizes his expected utility under the correct belief may be driven out by an agent who maximizes his expected utility under an incorrect belief, simply because the former does not maximize a logarithmic utility function, whereas the latter does. [1] were the first to show this implication of the Kelly criterion in a standard asset pricing model. As a result, the market selection hypothesis fails because agents with accurate beliefs are not selected. A consequence of this failure is that asset prices may not eventually reflect the beliefs of agents who make accurate predictions, and hence may persistently deviate from the rational expectations equilibrium and violate the efficient market hypothesis.

However, a series of recent studies indicates that the early analysis of [1] is not complete. [10] shows that, if the saving behavior is endogenously determined, then the market selection hypothesis is rescued, and in the long run, only those optimizing investors with correct beliefs survive. The surviving agents do not have to be log-utility maximizers, and they can have diverse risk preferences. [10]'s analysis is further confirmed by [2] in a connection of the market selection hypothesis to the first theorem of welfare economics. [2] show that in a dynamic and complete market Pareto optimality is the key to understanding selection either for or against traders with correct beliefs: in any optimal allocation the survival or disappearance of a trader is determined entirely by beliefs, and not by risk preferences.

Despite the rigorousness of these theoretical studies, there exists a fundamental limitation, which may make it difficult to grasp their empirical counterparts, namely, they are non-constructive. ${ }^{4}$ Take [10] as an example. First, the analysis crucially depends on the appearance of agents who eventually make accurate predictions or eventually make accurate next period predictions. Nevertheless, the process that shows the emergence of these sages is unknown. It is, therefore, not clear how these agents emerge, or whether they will ever emerge. ${ }^{5}$ Second, maximizing expected utility is equivalent to assuming that agents are able to solve any infinite-time stochastic dynamic optimization problem implied by their utility function. However, current dynamic optimization techniques, regardless of whether they include stochastic optimal control or stochastic dynamic programming, can only help us solve a very limited subset of the whole problem space. As for the rest of them, it is necessary to rely on numerical approximations, and their effectiveness to a large extent is also unknown.

Given these practical limitations, we are motivated to re-examine the issue from a more realistic perspective or, technically speaking, a computational perspective. By remaining in the general equilibrium analysis framework, we replace the rational agents with bounded-rational agents. More precisely, these agents are constructed in terms of what is known as autonomous agents in

\footnotetext{
${ }^{4}$ This kind of issue is generally shared in many general equilibrium analyses.

${ }^{5}$ Back to the real world, we have not been convinced that these agents have ever appeared in human history.
} 
agent-based computational economics ([12]). Basically, these agents are able to learn to optimize and to forecast in an autonomous manner. So, they are not necessarily utility-maximizers. Instead, they use adaptive computing techniques to approximate the optimal solution. In this sense, they are Herbert Simon's satisfying agents. Similarly, they base their decisions upon beliefs which may not be and may never be correct, but are reviewed and revised continuously ([9]).

By introducing autonomous agents, we are getting closer to the world of flesh and blood, and enhancing the study of the empirical relevance of risk preference to survival dynamics.

\section{A Simple Multi-Asset Model}

The simulations presented in this paper are based on an agent-based version of the multi-asset market as per the studies of [1] and [10]. The market is complete in the sense that the number of states is equal to the number of assets, say $M$. At each date $t$, the outstanding volume of each asset is exogenously fixed at one unit. There are $I$ investors in the market, with each being indexed by $i$. At time $t$ asset $m$ will pay dividends $w_{m}$ if the corresponding state $m$ occurs, and 0 otherwise. The behavior of these states follows a finitestate stochastic process, which does not have to be stationary. The dividends $w_{m}$ will be distributed among the $I$ investors proportionately according to their owned shares of the respective asset. The dividends can only be either re-invested or consumed. Hoarding is prohibited. If agent $i$ chooses to consume $c$, her satisfaction is measured by her utility function $u(c)$. This simple multi-asset market clearly defines an optimization problem for each individual as follows:

$$
\max _{\left\{\left\{\delta_{t+r}^{i}\right\}_{r=0}^{\infty},\left\{\alpha_{i+r}^{i}\right\}_{r=0}^{\infty}\right\}} E\left\{\sum_{r=0}^{\infty}\left(\beta^{i}\right)^{r} u^{i}\left(c_{t+r}^{i}\right) \mid B_{t-1}^{i}\right\}
$$

subject to

$$
\begin{array}{r}
c_{t+r}^{i}+\sum_{m=1}^{M} \alpha_{m, t+r}^{i, *} \cdot \delta_{t+r}^{i, *} \cdot W_{t+r-1}^{i} \leq W_{t+r-1}^{i} \quad \forall r \geq 0, \\
\sum_{m=1}^{M} \alpha_{m, t+r}^{i}=1, \quad \alpha_{m, t+r}^{i} \geq 0 \quad \forall r \geq 0 .
\end{array}
$$

In equation (1), $u^{i}$ is agent $i$ 's temporal utility function, and $\beta^{i}$, also called the discount factor, reveals agent $i$ 's time preference. The expectation $E()$ is taken with respect to the most recent belief $B_{t}^{i}$, which is a probabilistic model used to represent agent $i$ 's subjective belief regarding the stochastic nature of the state. The maximization problem asks for two sequences of decisions, one related to saving, and the other to the portfolios, denoted by 


$$
\left\{\left\{\delta_{t+r}^{i}\right\}_{r=0}^{\infty},\left\{\alpha_{t+r}^{i}\right\}_{r=0}^{\infty}\right\}
$$

where $\delta_{t}^{i}$ is the saving rate at time $t$, and

$$
\alpha_{t}^{i}=\left(\alpha_{1, t}^{i}, \alpha_{2, t}^{i}, \ldots, \alpha_{M, t}^{i}\right)
$$

is the portfolio comprising the $M$ assets.

Equations (2) and (3) are the budget constraints. $W_{t}^{i}$ is the wealth of agent $i$ at time $t$, which is earned from the dividends paid at time $t$. Notice that these budget constraints do not allow agents to consume or invest by borrowing.

The equilibrium price $\rho_{m, t}$ is determined by equating the demand for asset $m$ to the supply of asset $m$, i.e.

$$
\sum_{i=1}^{I} \frac{\alpha_{m, t}^{i, *} \cdot \delta_{t}^{i, *} \cdot W_{t-1}^{i}}{\rho_{m, t}}=1, \quad m=1,2, \ldots, M .
$$

By rearranging Equation (4), we obtain the market equilibrium price of asset $m$ :

$$
\rho_{m, t}=\sum_{i=1}^{I} \alpha_{m, t}^{i, *} \cdot \delta_{t}^{i, *} \cdot W_{t-1}^{i} .
$$

\section{The Agent-Based Multi-Asset Artificial Stock Market}

An agent-based version of the Blume-Easley-Sandroni standard multi-asset model is developed in [4]. There they ([4]) propose a sliding-window adaptation scheme to approximate the original infinite-time horizon optimization problem (Equations (1) - (3)) by a finite-time horizon optimization problem. The stochastic optimization problem (1) has two mainstays: first, finding an appropriate belief, and second, under that belief, searching for the best decisions regarding saving and portfolios. To distinguish the two, [3] calls the former "learning how to forecast," and the latter learning how to optimize. Genetic algorithms are then applied to evolve both beliefs and investment strategies. $^{6}$

To simulate this agent-based multi-asset artificial stock market, a software called AIE-ASM Version 5.0 is written using Delphi, Version 6.0. In each single run, we generate a series of artificial data. At the micro level, it includes the dynamics of agents' beliefs, investment behavior, and the associated wealth

$$
\left\{B_{t}^{i, *}, \delta_{t}^{i}, \alpha_{t}^{i}, W_{t}^{i}\right\}_{t=1}^{100}, \quad i=1, \ldots, I .
$$

At the aggregate level, we observe the asset price dynamics

$$
\left\{\rho_{m, t}\right\}_{t=1}^{100}, \quad m=1, \ldots, M .
$$

Figure 1 displays the time series plat of prices in a five-asset market. In this specific simulation, the state follows an i.i.d. process.

\footnotetext{
${ }^{6}$ Details can be found in [4].
} 


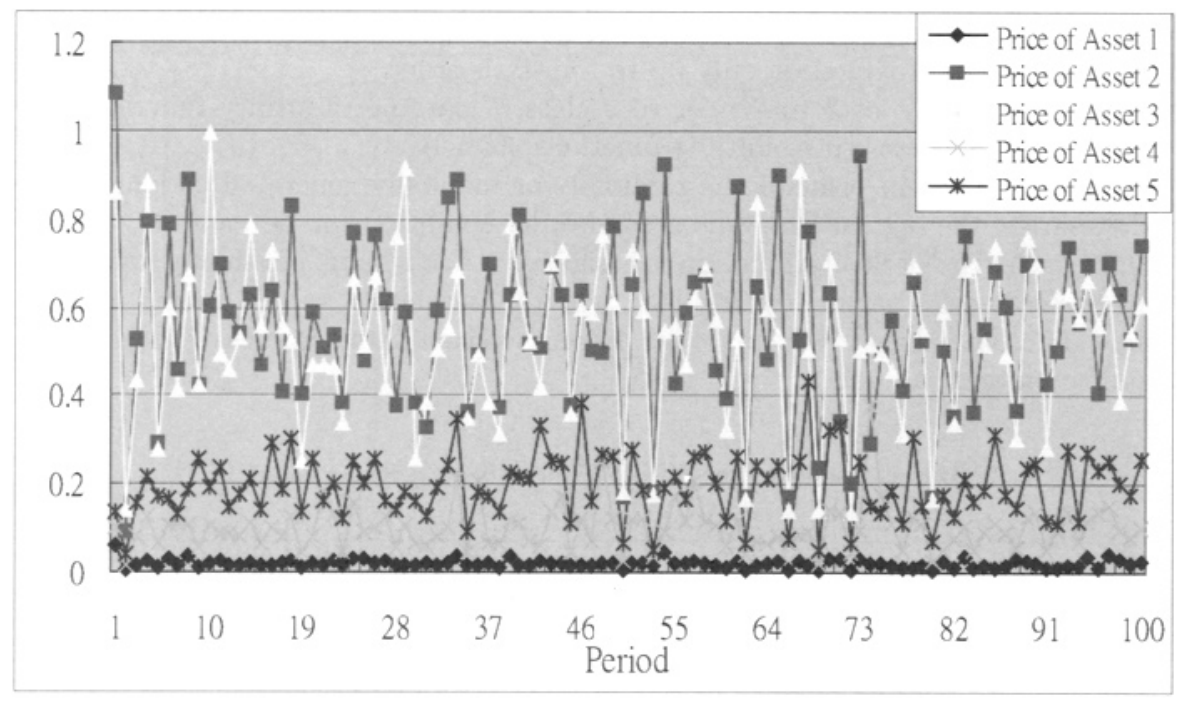

Fig. 1. Time Series Plot of the Prices of Assets; $M=5$.

By multiplying the dividends by the associated probabilities, we can obtain the expected dividends for each asset, and they are $0.004,0.419,0.373,0.069$ and 0.133 in this case. The mean prices taken over the entire sample periods are $0.0191,0.558$, $0.530,0.099$ and 0.196 , respectively. It can then be clearly seen that the prices do reflect the underlying dividends.

\section{Experimental Design}

Since the main focus of this paper is to examine the relevance of risk preference to survivability, we shall assume that the autonomous agents are identical in all aspects except in terms of their preferences over risk. With this assumption, we run two series of experiments. These two experiments differ in their constituent agent types. In Experiment 1, the market is composed of eight types of agents, and they are distributed evenly among 40 market participants, i.e. five agents for each type. These eight types of agents are agents with the seven utility functions specified in Table 1 plus the CAPM (capital asset pricing model) believers.

The type-one agent has the logarithmic utility function. We are very much interested in knowing whether this type of agent has any advantage over others in the long-run wealth share. As to types two to six, they are also frequently used in economic analysis. ${ }^{7}$ Among them, type four has the wellknown $C A R A$ (constant absolute risk aversion) utility function. In addition to these six familiar types of utility functions, we also consider any arbitrary utility function. By using Taylor's expansion, an arbitrary analytical utility

\footnotetext{
${ }^{7}$ See, for example, [6], pp. 27-33.
} 
function can be approximated by a finite-order polynomial function. Here, we consider the approximation only up to the sixth order.

Notice that types 3 to 7 refer to a class of parametric utility functions. Parameters of these types of utility functions, namely, $\alpha_{1}, \ldots, \alpha_{4}, \beta_{1}, \ldots, \beta_{3}$, and $a_{0}, a_{1}, \ldots, a_{6}$, can in principle be randomly or manually generated as long as they satisfy the regular first- and second-order conditions: $u^{\prime}>0$ and $u^{\prime \prime}<0$. Since each type of utility function is assigned to five agents, parameter values are generated for each agent for each type separately. So, type 3 agents may have different values of $\left(\alpha_{1}, \beta_{1}\right)$, type 4 agents have different values of $\left(\alpha_{2}, \beta_{2}\right)$, and so on and so forth.

Table 1. Types of the Utility Function $u(c)$ : Experiment 1

\begin{tabular}{|c|c|c|}
\hline & Utility Type & Relative Risk Aversion (RRA) \\
\hline Type 1 & $u(c)=\log (c)$ & 1 \\
\hline Type 2 & $u(c)=\sqrt{c}$ & 0.5 \\
\hline Type 3 & $u(c)=\alpha_{1}+\beta_{1} c$ & 0 \\
\hline Type 4 & $u(c)=\frac{\alpha_{2}}{\beta_{2}} \exp \left\{\beta_{2} c\right\}$ & $-\beta_{2} c$ \\
\hline Type 5 & $u(c)=\frac{1}{\left(\gamma_{3}+1\right) \beta_{3}}\left(\alpha_{3}+\beta_{3} c\right)^{\gamma_{3}+1}$ & $-\frac{\beta_{3} \gamma_{3}}{c}$ \\
\hline Type 6 & $u(c)=c-\frac{\alpha_{4}}{2} c^{2}$ & $\frac{\alpha_{3}}{\alpha_{4}}$ \\
\hline Type 7 & $u(c)=a_{0}+\sum_{i=1}^{6} a_{i} c^{i}$ & $-\frac{2 a_{2} c+6 a_{3} c^{2}+12 a_{4} c^{3}+20 a_{5} c^{4}+30 a_{6} c^{5}}{a_{1}+2 a_{2} c+3 a_{3} c^{2}+4 a_{4} c^{3}+5 a_{5} c^{4}+6 a_{6} c^{5}}$ \\
\hline
\end{tabular}

In Experiment 2, all agents are restricted to the family of the CRRA (constant relative risk aversion) utility functions,

$$
u(c)=\left\{\begin{array}{l}
c^{\rho} / \rho, \quad \text { if }-\infty<\rho<\infty, \\
\ln ^{c}, \quad \text { if } \rho=0 .
\end{array}\right.
$$

They, however, differ in terms of their RRA coefficients, i.e. $1-\rho$. The smaller the $\rho$, the larger the risk aversion coefficient. Eleven different $\rho$ s, starting from $0,0.1 ., 0.2, \ldots$, to 0.9 , and 1.0 , are distributed evenly to all 55 agents, with five agents for each $\rho$.

\section{Experimental Results}

Figure 2 shows the wealth-share dynamics of the eight types of investors in Experiment 1. Notice that each line is based on the average of 100 simulations. The results clearly indicate the strong dominance of the type-one investors, i.e. the agents who have a log utility function. While in some cases typetwo investors are still hanging in there for the first 100 periods, their shares eventually decline toward zero. Maybe the most striking result is the extinction of the CARA type of agents (type-4 agents). It is striking because the CARA 


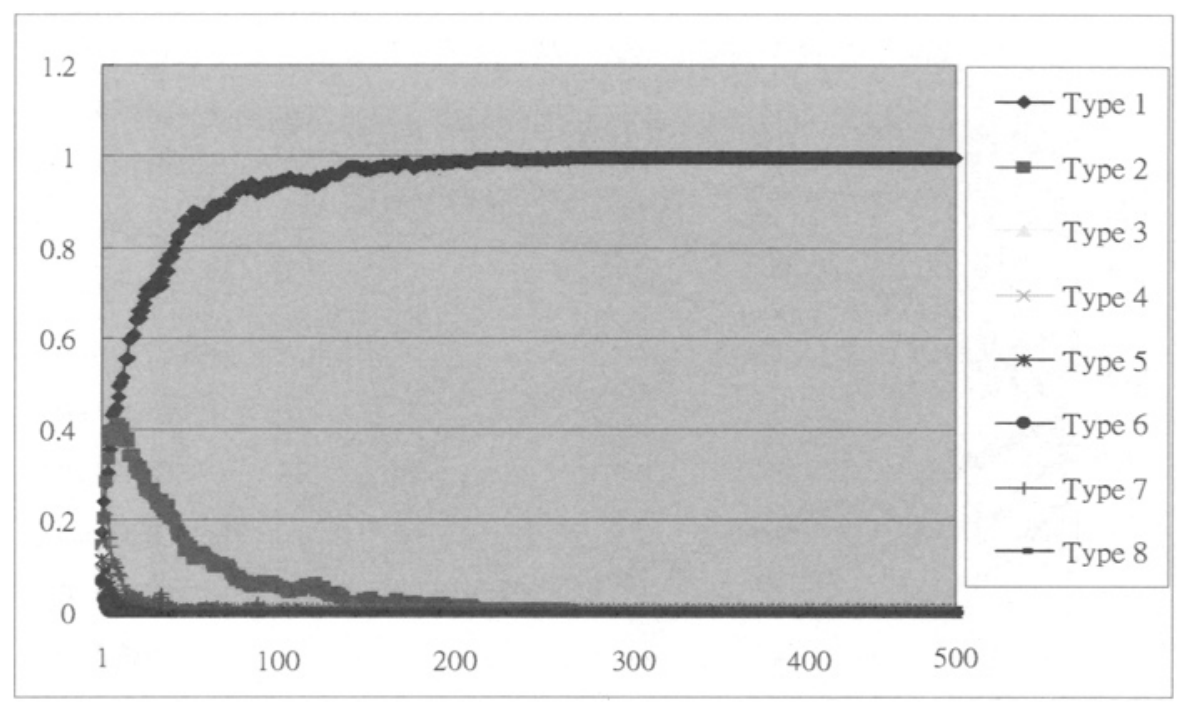

Fig. 2. Time Series Plot of the Wealth Share of Eight Types of Investors: Experiment 1

utility function has been used so extensively in the finance literature that one can hardly cast any doubt on its appropriateness. ${ }^{8}$ Equally surprising is the fiding that CAPM believers also fail to survive. This result is consistent with an earlier finding by [11], who shows that a sufficient condition to drive CAPM traders to extinction is that an investor endowed with a logarithmic utility function enters the market.

Since the type-one investors have a constant relative risk aversion coeffcient that is one, our experimental results also lend support to Blume and Easley's main argument: the market selects those investors whose coefficient of relative risk aversion is nearly one. ${ }^{9}$ To further examine this claim, the wealth share dynamics of Experiment 2 is depicted in Figure 3.

As can be seen from Figure 3, the wealth share seems to be positively correlated with the RRA coefficient. Agents with very low values for their the RRA coefficients are driven out of the market at different speeds. The lower the RRA, the faster the evaporation. Towards the end of this 100-period simulation, all agents with RRA values of less than 0.6 are driven out of the market. However, when the RRA coefficient increases to 0.9 , the respective

${ }^{8}$ For example, it was used to develop the standard asset pricing model ([5]), and was also used in agent-based artificial stock market simulations ([7]).

${ }^{9}$ See [1], Theorem 5.4, pp. 23-24. The words in italics shown in the main text are not quoted exactly from that theorem, which was originally made by controlling saving rates. Since saving rates are treated endogenously in our paper, our finding suggests that the theorem can still hold true even if the assumption of saving rates is relaxed. 


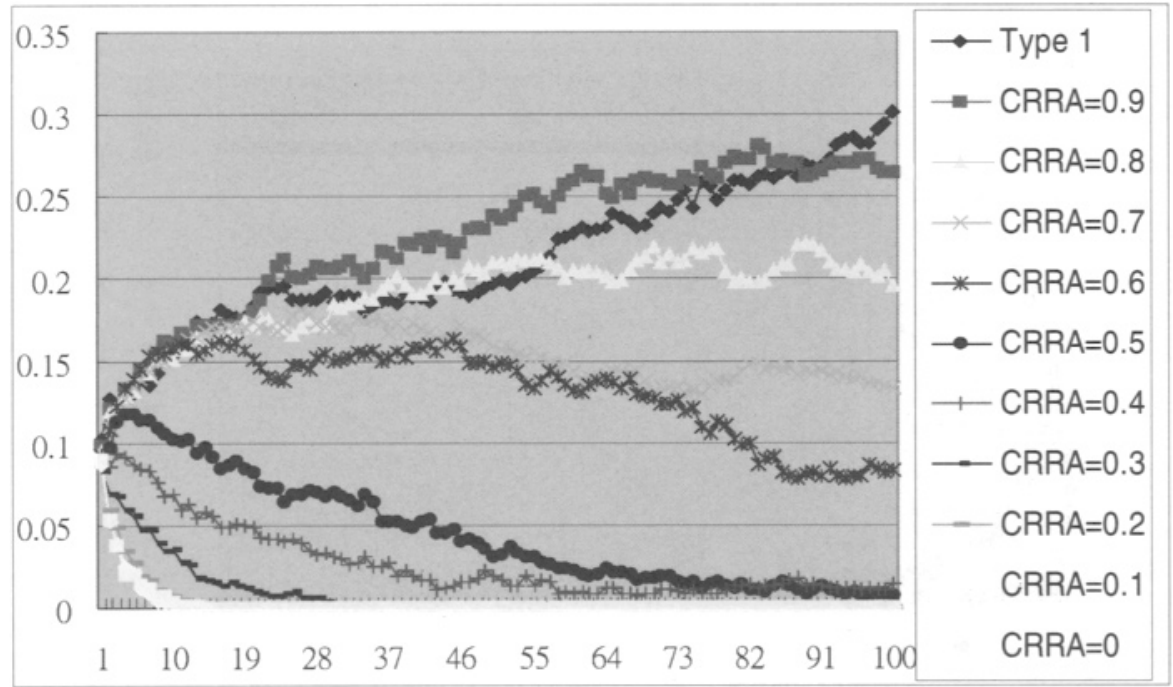

Fig. 3. Time Series Plot of the Wealth Shares of Eleven Types of Investors: Experiment 2

agents perform equally well, and sometimes even better, in terms of their wealth shares, as compared with the log-utility agents.

\section{Concluding Remarks}

The irrelevance of risk preference to the survivability of agents is dismissed in this paper. Our first experiment indicates that the only agents who survive in the long run (up to a 500-period simulation) are the log-utility agents. The rest are all driven out, including the CARA agents and the CAPM believers. In the second experiment, we further test for the significance of the RRA coefficient by assuming that all agents are CRRA types, and it is found that the agents' wealth share is affected by how close their RRA coefficients are to 1.

\section{Acknowledgements}

National Science Council (NSC) funding support NSC:91-2415-H-004-005 is greatly appreciated by the authors.

\section{References}

1. Blume E, Easley E (1992) Evolution and Market Behavior. Journal of Economic Theory 58: 9-40. 
2. Blume E, Easley E (2003) If You're So Smart, Why Aren't You Rich? Belief Selection in Complete and Incomplete Markets. Working paper.

3. Bullard J, Duffy J (1999) Using Genetic Algorithms to Model the Evolution of Heterogeneous Beliefs. Computational Economics 13 (1): 41-60.

4. Chen S-H, Huang Y-C (2004) Risk Preference, Forecasting Accuracy and Survival Dynamics: Simulations Based on a Multi-Asset Agent-Based Artificial Stock Market. Working Paper Series 2004-1, AI-ECON Research Center, National Chengchi University.

5. Grossman SJ, Stiglitz J (1980) On the Impossibility of Informationally Efficienct Markets. American Economic Review 70: 393-408.

6. Huang C-F, Litzenberger RH (1988) Foundations for Financial Economics. Prentice Hall, North-Holland New York.

7. Izumi K, Nakamura S, Ueda K (2004) Development of an Artificial Market Model Based on a Field Study. Information Sciences, forthcoming.

8. Kelly JL (1956) A New Interpretatatin of Information Rate. Bell System Technical Journal 35: 917-926.

9. Lucas R (1986) Adaptive Behaviour and Economic Theory. In: Hogarth R, Reder M (eds) Rational Choice: The Contrast between Economics and Psychology. University of Chicago Press: 217-242.

10. Sandroni A (2000) Do Markets Favor Agents Able to Make Accurate Predictions? Econometrica 68: 1303-1341.

11. Sciubba E (1999) The Evolution of Portfolio Rules and the Capital Asset Pricing Model. DAE Working Paper No. 9909, University of Cambridge.

12. Tesfatsion L (2001) Introduction to the Special Issue on Agent-Based Computational Economics. Journal of Economic Dynamics and Control 25: 281-293. 